Bull. Korean Math. Soc. 52 (2015), No. 3, pp. 741-749

http://dx.doi.org/10.4134/BKMS.2015.52.3.741

\title{
THE $n$-TH TWISTED CHANGHEE POLYNOMIALS AND NUMBERS
}

\author{
Seog-Hoon Rim, Jin-Woo Park, Sung-Soo Pyo, and Jongkyum Kwon
}

\begin{abstract}
The Changhee polynomials and numbers are introduced in [6]. Some interesting identities and properties of those polynomials are derived from umbral calculus (see [6]). In this paper, we consider Witttype formula for the $n$-th twisted Changhee numbers and polynomials and derive some new interesting identities and properties of those polynomials and numbers from the Witt-type formula which are related to special polynomials.
\end{abstract}

\section{Introduction}

Let $p$ be an odd prime number. $\mathbb{Z}_{p}, \mathbb{Q}_{p}$ and $\mathbb{C}_{p}$ will denote the ring of $p$-adic integers, the field of $p$-adic numbers and the completion of algebraic closure of $\mathbb{Q}_{p}$. The $p$-adic norm $|\cdot|_{p}$ is normalized by $|p|_{p}=\frac{1}{p}$. Let $\mathbb{C}\left(\mathbb{Z}_{p}\right)$ be the space of continuous functions on $\mathbb{Z}_{p}$. For $f \in \mathbb{C}\left(\mathbb{Z}_{p}\right)$, the fermionic $p$-adic integral on $\mathbb{Z}_{p}$ is defined by Kim to be

$$
I_{-1}(f)=\int_{\mathbb{Z}_{p}} f(x) d \mu_{-1}(x)=\lim _{N \rightarrow \infty} \sum_{x=0}^{p^{N}-1} f(x)(-1)^{x}, \quad(\text { see }[8,15]) .
$$

Let $f_{1}(x)=f(x+1)$. Then, by (1.1), we get

$$
I_{-1}\left(f_{1}\right)+I_{-1}(f)=2 f(0), \quad(\text { see }[3,5,7-11]) .
$$

It is well known that the Euler polynomials are defined by the generating function to be

$$
\frac{2}{\varepsilon e^{t}+1} e^{x t}=\sum_{n=0}^{\infty} E_{n, \varepsilon}(x) \frac{t^{n}}{n !},
$$

(see $[1-14,16])$.

Received November 6, 2013; Revised February 5, 2014.

2010 Mathematics Subject Classification. 11S80, 11B68, 05A30.

Key words and phrases. Euler numbers, Changhee numbers, twisted Changhee numbers. 
When $x=0, E_{n, \varepsilon}=E_{n, \varepsilon}(0)$ are called the $n$-th twisted Euler numbers. The Changhee polynomials are defined by the generating function to be

$$
\frac{2}{t+2}(1+t)^{x}=\sum_{n=0}^{\infty} C h_{n}(x) \frac{t^{n}}{n !}, \quad(\text { see }[6]) .
$$

When $x=0, C h_{n}=C h_{n}(0)$ are called the Changhee numbers, (see [6]).

The Stirling number of the first kind is defined by

$$
(x)_{n}=x(x-1) \cdots(x-n+1)=\sum_{l=0}^{n} S_{1}(n, l) x^{l}, \quad(\text { see }[6]) .
$$

The Changhee numbers and polynomials are introduced in [9]. Many interesting identities of those numbers and polynomials arise from umbral calculus (see [9]). We consider Witt-type formula for the $n$-th twisted Changhee numbers and polynomials and derive some new interesting identities and properties of those polynomials and numbers from the Witt-type formula which are related to special polynomials.

\section{The $n$-th twisted Changhee numbers and polynomials}

For $n \in \mathbb{N}$, let $T_{p}$ be the $p$-adic locally constant space defined by

$$
T_{p}=\bigcup_{n \geq 1} \mathbb{C}_{p^{n}}=\lim _{n \rightarrow \infty} \mathbb{C}_{p^{n}}
$$

where $\mathbb{C}_{p^{n}}=\left\{\omega \mid \omega^{p^{n}}=1\right\}$ is the cyclic group of order $p^{n}$.

For $\varepsilon \in T_{p}$, the $n$-th twisted changhee polynomial are defined by

$$
\begin{aligned}
\sum_{n=0}^{\infty} C h_{n, \varepsilon}(x) \frac{t^{n}}{n !} & =\int_{\mathbb{Z}_{p}}(1+\varepsilon t)^{x+y} d \mu_{-1}(y) \\
& =(1+\varepsilon t)^{x} \int_{\mathbb{Z}_{p}}(1+\varepsilon t)^{y} d \mu_{-1}(y) \\
& =\frac{2}{2+\varepsilon t}(1+\varepsilon t)^{x}
\end{aligned}
$$

and

$$
\begin{aligned}
\int_{\mathbb{Z}_{p}}(1+\varepsilon t)^{x+y} d \mu_{-1}(y) & =\sum_{n=0}^{\infty} \varepsilon^{n} \int_{\mathbb{Z}_{p}}\left(\begin{array}{c}
x+y \\
n
\end{array}\right) d \mu_{-1}(y) t^{n} \\
& =\sum_{n=0}^{\infty} \varepsilon^{n} \int_{\mathbb{Z}_{p}}(x+y)_{n} d \mu_{-1}(y) \frac{t^{n}}{n !} .
\end{aligned}
$$

Therefore, by (2.1) and (2.2) we obtained the following theorem.

Theorem 2.1. For $n \geq 0$, we have

$$
\varepsilon^{n} \int_{\mathbb{Z}_{p}}(x)_{n} d \mu_{-1}(x)=C h_{n, \varepsilon},
$$

where $C h_{n, \varepsilon}$ are called the $n$-th twisted Changhee numbers. 
Therefore, by (2.1) and (2.2), we obtain the following theorem.

Theorem 2.2. For $n \geq 0$, we have

$$
\varepsilon^{n} \int_{\mathbb{Z}_{p}}(x+y)_{n} d \mu_{-1}(y)=C h_{n, \varepsilon}(x) .
$$

From (2.1) and (2.2), we note that

$$
\sum_{n=0}^{\infty} \varepsilon^{n} \int_{\mathbb{Z}_{p}}\left(\begin{array}{l}
x \\
n
\end{array}\right) d \mu_{-1}(x) t^{n}=\frac{2}{2+\varepsilon t}=\sum_{n=0}^{\infty}\left(-\frac{\varepsilon}{2}\right)^{n} t^{n}
$$

Thus, by comparing the coefficients on the both sides, we obtain the following theorem.

Theorem 2.3. For $n \geq 0$, we have

$$
\int_{\mathbb{Z}_{p}}\left(\begin{array}{l}
x \\
n
\end{array}\right) d \mu_{-1}(x)=(-1)^{n}\left(\frac{1}{2}\right)^{n}, \quad(\text { see }[6]) .
$$

Replacing $t$ by $e^{t}-\frac{1}{\varepsilon}$ in (1.3), we get

$$
\varepsilon^{x} \sum_{n=0}^{\infty} E_{n, \varepsilon}(x) \frac{t^{n}}{n !}=\frac{2}{\varepsilon e^{t}+1}\left(\varepsilon e^{t}\right)^{x}=\sum_{n=0}^{\infty} C h_{n, \varepsilon}(x) \frac{1}{n !}\left(e^{x}-\frac{1}{\varepsilon}\right)^{n},
$$

where $E_{n, \varepsilon}(x)$ is the $n$-th twisted Euler polynomials and

$$
\begin{aligned}
\sum_{n=0}^{\infty} C h_{n, \varepsilon}(x) \frac{1}{n !}\left(e^{x}-\frac{1}{\varepsilon}\right)^{n} & =\sum_{n=0}^{\infty} C h_{n, \varepsilon}(x) \frac{1}{n !} \varepsilon^{-n} n !\left(\sum_{m=n}^{\infty} S_{2}(m, n) \frac{t^{m}}{m !}\right) \\
& =\sum_{m=0}^{\infty} \sum_{n=0}^{m} C h_{n, \varepsilon}(x) S_{2}(m, n) \varepsilon^{-n} \frac{t^{n}}{m !} .
\end{aligned}
$$

By comparing the coefficients on the both sides of (2.4) and (2.5), we obtain the following theorem.

Theorem 2.4. For $m \geq 0$, we have

$$
E_{m, \varepsilon}=\sum_{n=0}^{m} \varepsilon^{-n-x} C h_{m, \varepsilon} S_{2}(m, n),
$$

where $S_{2}(m, n)$ is the Stirling number of the second kind.

By Theorem 2.2, we easily get

$$
\begin{aligned}
C h_{n, \varepsilon}(x) & =\varepsilon^{n} \int_{\mathbb{Z}_{p}}(x+y)_{n} d \mu_{-1}(y) \\
& =\varepsilon^{n} \sum_{l=0}^{n} S_{1}(n, l) \int_{\mathbb{Z}_{p}}(x+y)^{l} d \mu_{-1}(y) \\
& =\varepsilon^{n} \sum_{l=0}^{n} S_{1}(n, l) E_{l}(x)
\end{aligned}
$$


Therefore, by (2.6), we obtain the following theorem.

Theorem 2.5. For $n \geq 0$, we have

$$
C h_{n, \varepsilon}(x)=\varepsilon^{n} \sum_{l=0}^{n} S_{1}(n, l) E_{l}(x),
$$

where $S_{1}(n, l)$ is the Stirling number of the first kind.

The $n$-th twisted Changhee polynomials of order $k$ are defined by the generating function to be

$$
\begin{aligned}
& \sum_{n=0}^{\infty} C h_{n, \varepsilon}^{(k)}(x) \frac{t^{n}}{n !} \\
= & \int_{\mathbb{Z}_{p}} \cdots \int_{\mathbb{Z}_{p}}(1+\varepsilon t)^{x_{1}+x_{2}+\cdots+x_{k}+x} d \mu_{-1}\left(x_{1}\right) \cdots d \mu_{-1}\left(x_{k}\right) \\
= & (1+\varepsilon t)^{x} \int_{\mathbb{Z}_{p}} \cdots \int_{\mathbb{Z}_{p}}(1+\varepsilon t)^{x_{1}+x_{2}+\cdots+x_{k}} d \mu_{-1}\left(x_{1}\right) \cdots d \mu_{-1}\left(x_{k}\right) \\
= & (1+\varepsilon t)^{x}\left(\frac{2}{1+\varepsilon t}\right)^{k} .
\end{aligned}
$$

We observe that

$$
\begin{aligned}
& \int_{\mathbb{Z}_{p}} \cdots \int_{\mathbb{Z}_{p}}(1+\varepsilon t)^{x_{1}+x_{2}+\cdots+x_{k}+x} d \mu_{-1}\left(x_{1}\right) \cdots d \mu_{-1}\left(x_{k}\right) \\
= & \sum_{n=0}^{\infty} \varepsilon^{n} \int_{\mathbb{Z}_{p}}\left(x_{1}+x_{2}+\cdots+x_{k}+x\right)_{n} d \mu_{-1}\left(x_{1}\right) \cdots d \mu_{-1}\left(x_{k}\right) \frac{t^{n}}{n !} .
\end{aligned}
$$

Therefore, by (2.7), we obtain the generating function of $C h_{n, \varepsilon}^{(k)}$ as follows.

Theorem 2.6. The generating function of $C h_{n, \varepsilon}^{(k)}$ is given by

$$
\sum_{n=0}^{\infty} C h_{n, \varepsilon}^{(k)} \frac{t^{n}}{n !}=\int_{\mathbb{Z}_{p}} \cdots \int_{\mathbb{Z}_{p}}(1+\varepsilon t)^{x_{1}+x_{2}+\cdots+x_{k}} d \mu_{-1}\left(x_{1}\right) \cdots d \mu_{-1}\left(x_{k}\right) .
$$

From (2.8), we have

$$
\varepsilon^{n} \int_{\mathbb{Z}_{p}} \cdots \int_{\mathbb{Z}_{p}}\left(\begin{array}{c}
x_{1}+x_{2}+\cdots+x_{k} \\
n
\end{array}\right) d \mu_{-1}\left(x_{1}\right) \cdots d \mu_{-1}\left(x_{k}\right)=\frac{C h_{n, \varepsilon}^{(k)}}{n !} .
$$

By (2.6), we get

$$
\begin{aligned}
C h_{n, \varepsilon}^{(k)} & =\varepsilon^{n} \sum_{l=0}^{n} S_{1}(n, l) \int_{\mathbb{Z}_{p}} \cdots \int_{\mathbb{Z}_{p}} x_{1}^{l} \cdots x_{k}^{l} d \mu_{-1}\left(x_{1}\right) \cdots d \mu_{-1}\left(x_{k}\right) \\
& =\varepsilon^{n} \sum_{l=0}^{n} S_{1}(n, l)\left(E_{l}\right)^{k}
\end{aligned}
$$


where $\left(E_{l}\right)^{k}=\underbrace{E_{l} \times E_{l} \times \cdots \times E_{l}}_{k \text {-times }}$.

Therefore, by (2.10), we obtain the following theorem.

Theorem 2.7. For $n \geq 0, k \geq 1$, we have

$$
C h_{n, \varepsilon}^{(k)}=\varepsilon^{n} \sum_{l=0}^{n} S_{1}(n, l)\left(E_{l}\right)^{k},
$$

where $S_{1}(m, n)$ is the Stirling number of the first kind.

Now we consider the $n$-th twisted Changhee polynomials of the second kind as follows:

$$
\begin{aligned}
\sum_{n=0}^{\infty} \widehat{C h}_{n, \varepsilon}(x) \frac{t^{n}}{n !} & =\int_{\mathbb{Z}_{p}}(1+\varepsilon t)^{-y+x} d \mu_{-1}(y) \\
& =(1+\varepsilon t)^{x} \int_{\mathbb{Z}_{p}}(1+\varepsilon t)^{-y} d \mu_{-1}(y) \\
& =\frac{2(1+\varepsilon t)}{2+\varepsilon t}(1+\varepsilon t)^{x}
\end{aligned}
$$

Hence,

$$
\begin{aligned}
\widehat{C h}_{n, \varepsilon}(x) & =\varepsilon^{n} \int_{\mathbb{Z}_{p}}(-y+x)_{n} d \mu_{-1}(y) \\
& =\varepsilon^{n} \sum_{l=0}^{n} S_{1}(n, l)(-1)^{l} \int_{\mathbb{Z}_{p}}(y-x)^{l} d \mu_{-1}(y) \\
& =\varepsilon^{n} \sum_{l=0}^{n} S_{1}(n, l)(-1)^{l} E_{l}(-x) .
\end{aligned}
$$

Therefore, by (2.12), we obtain the following theorem.

Theorem 2.8. For $n \geq 0$, we have

$$
\widehat{C h}_{n, \varepsilon}(x)=\varepsilon^{n} \sum_{l=0}^{n} S_{1}(n, l)(-1)^{l} E_{l}(-x) .
$$

By replacing $t$ by $e^{t}-\frac{1}{\varepsilon}$ in $(2.11)$

$$
\begin{aligned}
& \sum_{m=0}^{\infty} \sum_{n=0}^{m} \widehat{C h}_{n, \varepsilon}(x) \varepsilon^{-n} S_{2}(m, n) \frac{t^{m}}{m !} \\
= & \sum_{n=0}^{\infty} \widehat{C h}_{n, \varepsilon}(x) \frac{1}{n !} n ! \varepsilon^{-n} \sum_{m=n}^{\infty} S_{2}(m, n) \frac{t^{m}}{m !} \\
= & \sum_{n=0}^{\infty} \widehat{C h}_{n, \varepsilon}(x) \frac{1}{n !}\left(e^{t}-\frac{1}{\varepsilon}\right)^{n}
\end{aligned}
$$




$$
\begin{aligned}
& =\frac{2}{\varepsilon e^{t}+1}\left(\varepsilon e^{t}\right)^{x+1} \\
& =\varepsilon^{x+1} \frac{2}{\varepsilon e^{t}+1} e^{t(x+1)} \\
& =\varepsilon^{x+1} \sum_{m=0}^{\infty} E_{m, \varepsilon}(x+1) \frac{t^{m}}{m !} .
\end{aligned}
$$

Therefore, by (2.13), we obtain the following theorem.

Theorem 2.9. For $m \geq 0$, we have

$$
E_{m}(x+1)=\sum_{n=0}^{m} \widehat{C h}_{n, \varepsilon}(x) \varepsilon^{-n-x-1} S_{2}(m, n) .
$$

Now, we consider the $n$-th twisted Changhee polynomials of the first kind relate to the $n$-th twisted Changhee polynomials of the second kind.

$$
\begin{aligned}
\frac{(-1)^{n} C h_{n, \varepsilon}(x)}{n !} & =(-1)^{n} \varepsilon^{n} \int_{\mathbb{Z}_{p}}\left(\begin{array}{c}
x+y \\
n
\end{array}\right) d \mu_{-1}(y) \\
& =\varepsilon^{n} \int_{\mathbb{Z}_{p}}\left(\begin{array}{c}
-x-y+n-1 \\
n
\end{array}\right) d \mu_{-1}(y) \\
& =\varepsilon^{n} \sum_{m=0}^{\infty}\left(\begin{array}{c}
n-1 \\
n-m
\end{array}\right) \int_{\mathbb{Z}_{p}}\left(\begin{array}{c}
-x-y \\
m
\end{array}\right) d \mu_{-1}(y) \\
& =\varepsilon^{n} \sum_{m=1}^{n}\left(\begin{array}{c}
n-1 \\
m-1
\end{array}\right) \varepsilon^{-m} m ! \varepsilon^{m} \int_{\mathbb{Z}_{p}}\left(\begin{array}{c}
-x-y \\
m
\end{array}\right) d \mu_{-1}(y) \\
& =\sum_{m=1}^{n}\left(\begin{array}{c}
n-1 \\
m-1
\end{array}\right) \varepsilon^{n-m} \frac{\widehat{C h_{m, \varepsilon}}(-x)}{m !}
\end{aligned}
$$

Therefore, for $n \geq 1$, we have

$$
\frac{(-1) C h_{n, \varepsilon}(x)}{n !}=\sum_{m=1}^{n}\left(\begin{array}{c}
n-1 \\
m-1
\end{array}\right) \varepsilon^{n-m} \frac{\widehat{C h}_{m, \varepsilon}(-x)}{m !} .
$$

For $k \in \mathbb{N}$, let us consider the $n$-th twisted Changhee numbers of order $k$ as follows:

$$
\varepsilon^{n} \int_{\mathbb{Z}_{p}} \cdots \int_{\mathbb{Z}_{p}}\left(-x_{1}-x_{2} \cdots-x_{k}\right)_{n} d \mu_{-1}\left(x_{1}\right) \cdots d \mu_{-1}\left(x_{k}\right)=\widehat{C h}_{n, \varepsilon},
$$

where $n \in \mathbb{Z}_{\geq 0}$.

Then the generating function of $\widehat{C h}_{n, \varepsilon}^{(k)}$ is given by

$$
\begin{aligned}
& \sum_{n=0}^{\infty} \widehat{C h}_{n, \varepsilon}^{(k)} \frac{t^{n}}{n !} \\
= & \int_{\mathbb{Z}_{p}} \cdots \int_{\mathbb{Z}_{p}}(1+\varepsilon t)^{-x_{1}-\cdots-x_{k}} d \mu_{-1}\left(x_{1}\right) \cdots d \mu_{-1}\left(x_{k}\right)
\end{aligned}
$$




$$
=\sum_{n=0}^{\infty} \varepsilon^{n} \int_{\mathbb{Z}_{p}} \cdots \int_{\mathbb{Z}_{p}}\left(-x_{1}-\cdots-x_{k}\right)_{n} d \mu_{-1}\left(x_{1}\right) \cdots d \mu_{-1}\left(x_{k}\right) \frac{t^{n}}{n !} .
$$

Now, we observe that

(2.19)

$$
\begin{aligned}
& \int_{\mathbb{Z}_{p}} \cdots \int_{\mathbb{Z}_{p}}(1+\varepsilon t)^{-x_{1}-\cdots-x_{k}} d \mu_{-1}\left(x_{1}\right) \cdots d \mu_{-1}\left(x_{k}\right) \\
= & \sum_{m=0}^{\infty} \varepsilon^{m} \int_{\mathbb{Z}_{p}} \cdots \int_{\mathbb{Z}_{p}}(-1)^{m}\left(x_{1}+\cdots+x_{k}\right)^{m} d \mu_{-1}\left(x_{1}\right) \cdots d \mu_{-1}\left(x_{n}\right) \frac{(\log (1+\varepsilon t))^{m}}{m !} \\
= & \sum_{m=0}^{\infty} \varepsilon^{m}(-1)^{m}\left(E_{m}\right)^{k} \sum_{n=m}^{\infty} S_{1}(n, m) \frac{t^{n}}{n !} \\
= & \sum_{n=0}^{\infty}\left(\sum_{m=0}^{n} \varepsilon^{m}(-1)^{m}\left(E_{m}\right)^{k} S_{1}(n, m)\right) \frac{t^{n}}{n !} .
\end{aligned}
$$

From (2.18) and (2.19), we have

$$
\widehat{C h}_{n, \varepsilon}^{(k)}=\varepsilon^{n} \sum_{m=0}^{n}(-1)^{m}\left(E_{m}\right)^{k} S_{1}(n, m),
$$

where $\left(E_{m}\right)^{k}=\underbrace{E_{m} \times \cdots \times E_{m}}_{k \text {-times }}$.

Now, we define the $n$-th twisted Changhee polynomials of the second kind of order $k$ as follows:

$$
\widehat{C h}_{n, \varepsilon}^{(k)}(x)=\varepsilon^{n} \int_{\mathbb{Z}_{p}} \cdots \int_{\mathbb{Z}_{p}}\left(-x_{1} \cdots x_{k}-x\right)_{n} d \mu_{-1}\left(x_{1}\right) \cdots d \mu_{-1}\left(x_{k}\right) .
$$

Then the generating function of $\widehat{C h}_{n, \varepsilon}^{(k)}(x)$ are given by

$$
\begin{aligned}
& \sum_{n=0}^{\infty}{\widehat{C h_{n}}}^{(k)}(x) \frac{t^{n}}{n !} \\
= & \sum_{n=0}^{\infty} \int_{\mathbb{Z}_{p}} \cdots \int_{\mathbb{Z}_{p}} \varepsilon^{n}\left(-x_{1}-\cdots-x_{k}-x\right)_{n} d \mu_{-1}\left(x_{1}\right) \cdots d \mu_{-1}\left(x_{k}\right) \frac{t^{n}}{n !} \\
= & \int_{\mathbb{Z}_{p}} \cdots \int_{\mathbb{Z}_{p}}(1+\varepsilon t)^{-x_{1} \cdots x_{k}-x} d \mu_{-1}\left(x_{1}\right) \cdots d \mu_{-1}\left(x_{k}\right) .
\end{aligned}
$$

Proceeding similarly to (2.12), we have

$$
\widehat{C h}_{n, \varepsilon}^{(k)}(x)=\varepsilon^{n} \sum_{m=0}^{n}(-1)^{m} S_{1}(n, m) \sum_{l=0}^{m}\left(\begin{array}{c}
m \\
l
\end{array}\right)\left(E_{m-l}\right)^{k} x^{l},
$$

where $\left(E_{m-l}\right)^{k}=\underbrace{E_{m-l} \times \cdots \times E_{m-l}}_{k \text {-times }}$. 


\section{References}

[1] S. Araci and M. Acikgoz, A note on the Frobenius-Euler numbers and polynomials associated with Bernstein polynomials, Adv. Stud. Contemp. Math. 22 (2012), no. 3, 399-406.

[2] A. Bayad, Modular properties of elliptic Bernoulli and Euler functions, Adv. Stud. Contemp. Math. 20 (2010), no. 3, 389-401.

[3] J. Choi, D. S. Kim, T. Kim, and Y. H. Kim, Some arithmetic identities on Bernoulli and Euler numbers arising from the $p$-adic integrals on $\mathbb{Z}_{p}$, Adv. Stud. Contemp. Math. 22 (2012), no. 2, 239-247.

[4] D. Ding and J. Yang, Some identities related to the Apostol-Euler and Apostol-Bernoulli polynomials, Adv. Stud. Contemp. Math. 20 (2010), no. 1, 7-21.

[5] D. S. Kim, T. Kim, Y. H. Kim, and D. V. Dolgy, A note on Eulerian polynomials associated with Bernoulli and Euler numbers and polynomials, Adv. Stud. Contemp. Math. 22 (2012), no. 3, 379-389.

[6] D. S. Kim, T. Kim, and J. J. Seo, A note on Changhee polynomials and numbers, Adv. Studies Theor. Phys. 7 (2013), no. 20, 993-1003.

[7] T. Kim, Non-Archimedean q-integrals associated with multiple Changhee q-Bernoulli polynomials, Russ. J. Math. Phys. 10 (2003), no. 1, 91-98.

[8] , p-adic q-integrals associated with the Changhee-Barnes' $q$-Bernoulli polynomials, Integral Transforms Spec. Funct. 15 (2004), no. 5, 415-420.

[9] T. Kim, D. S. Kim, T. Mansour, S.-H. Rim, M. and Schork Umbral calculus and Sheffer sequences of polynomials, J. Math. Phys. 54 (2013), no. 8, 083504, 15 pp.

[10] T. Kim and S.-H. Rim, On Changhee-Barnes' q-Euler numbers and polynomials, Adv. Stud. Contemp. Math. 9 (2004), no. 2, 81-86.

[11] New Changhee q-Euler numbers and polynomials associated with p-adic qintegrals, Comput. Math. Appl. 54 (2007), no. 4, 484-489.

[12] Q.-M. Luo, q-analogues of some results for the Apostol-Euler polynomials, Adv. Stud. Contemp. Math. 20 (2010), no. 1, 103-113.

[13] C. S. Ryoo, T. Kim, and R. P. Agarwal, Exploring the multiple Changhee q-Bernoulli polynomials, Int. J. Comput. Math. 82 (2005), no. 4, 483-493.

[14] C. S. Ryoo and H. Song, On the real roots of the Changhee-Barnes' $q$-Bernoulli polynomials, Proceedings of the 15th International Conference of the Jangjeon Mathematical Society, 63-85, Jangjeon Math. Soc., Hapcheon, 2004.

[15] Y. Simsek, Special functions related to Dedekind-type DC-sums and their applications, Russ. J. Math. Phys. 17 (2010), no. 4, 495-508.

[16] Y. Simsek, T. Kim, and I. S. Pyung, Barnes' type multiple Changhee q-zeta functions, Adv. Stud. Contemp. Math. 10 (2005), no. 2, 121-129.

SEOG-HOON RIM

Department of Mathematics Education

KYUNGPOOK NATIONAL UNIVERSiTy

DAEGU 702-701, Korea

E-mail address: shrim@knu.ac.kr

JIN-WOO PARK

Department of Mathematics Education

DAEGU UNIVERSITY

Gyeongsan 712-714, KoreA

E-mail address: a0417001@knu.ac.kr 
Sung-SoO PyO

Department of Mathematics Education

KyUnGPOOK NATIONAL University

DAEGU 702-701, KorEA

E-mail address: ssoopyo@knu.ac.kr

JONGKYUM KWON

Department of Mathematics

KyungPoOK NATIONAL University

DAEGU 702-701, KorEA

E-mail address: mathkjk26@hanmail.net 